\title{
Editorial
}

\section{Sociedad 5.0 y \\ tecnologías \\ emergentes al 2030}

DOI: 10.29236/sistemas.n154a1

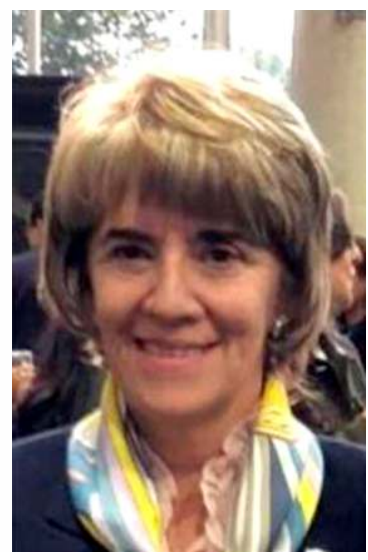

El ser humano como principal actor de la Sociedad 5.0, y las tecnologías emergentes a su servicio.

\section{María Mercedes Corral S.}

Llama la atención que la cuarta revolución industrial con toda la tecSegún Mark Vidal, prestigioso consultor en transformación, la quinta nología transformadora, con todos los avances y beneficios para la sociedad ahora se transforma en la quinta revolución, con el ser humano como protagonista y la tecnología a su servicio y de la sociedad. revolución industrial es la era de la humanidad y en su último libro, "La era de la humanidad hacia la quinta revolución industrial" defiende que, «a pesar de la inclusión absoluta de la tecnología en nuestras vidas, 
tendremos la oportunidad de ser más humanos que nunca». Esta es la oportunidad de cambio de la sociedad en lo económico, social y educativo, entre otros aspectos.

La presente edición gira alrededor de la Sociedad 5.0 y las tecnologías emergentes a 2030. Junto con un grupo de profesionales y expertos en temas de sociedad y tecnología fue posible aproximarnos a esta quinta revolución de la sociedad.

En primer lugar, tenemos una interesante entrevista con Orlando Ayala, líder reconocido internacionalmente en el área de las Tecnologías de la Información, profesional dedicado a estos temas y a la sociedad. En sus respuestas nos deja frases como "Es extremadamente urgente una sociedad en la que se humanice el ser humano y para lograrlo, entra en juego la redefinición de valores humanos, considerando lo que se puede hacer con la tecnología". Mediante estas reflexiones, Orlando reenfoca el tema que nos ocupa y a lo que en realidad está sucediendo hoy en la sociedad. Y deja el siguiente interrogante flotando en el ambiente. "¿siendo la tecnología una herramienta que no tiene corazón, alma o conciencia, se puede utilizar solamente en procura del bien de la humanidad?".

Por su parte, Julio Ernesto Rojas, antropólogo y doctor en Teoría de la Educación y Pedagogía Social, con 20 años de docencia universitaria, desde su perspectiva nos presenta cómo la tecnología se ha convertido en una necesidad, desde lo económico, social, cultural y lo político. Además, se refiere a la Sociedad 5.0 como un nuevo contrato social, en el que las tecnologías son los actores de cambio y las personas partícipes en el diálogo con estas tecnologías.

Nuestra columnista invitada es Laura Cortés-Rico, profesora auxiliar de Ingeniería en Multimedia de la Universidad Militar Nueva Granada. Su área de investigación es la de Interacciones Humano Computador, en particular en relación con tecnologías plurales que consideran aspectos técnicos, humanos y sociales. Ella aborda los desafíos que traerá la transición de la sociedad de la información, a la sociedad 5.0 del ser humano. Así mismo, señala como otro de los desafíos, la educación y la formación de los maestros; al que suma las estrategias sostenibles para toda la población y la localidad frente a la globalización.

Para este número de nuestra publicación, también se realizó una investigación sobre tendencias tecnológicas a nivel nacional, con utilización y conocimiento de tecnologías emergentes a 2030, a cargo de Emir Pernet, PhD, estudio que nos dará una idea sobre lo que está sucediendo alrededor de estas tecnologías, y cómo será el apoyo de la sociedad en el 2030. Una investigación similar se realizó en 2015 , 
base para la trazabilidad realizada con el propósito de observar la evolución en estos cinco años.

En el espacio tradicional del foro para la sección Cara y Sello, se contó con la participación de profesionales del sector privado y con investigadores de diferentes universidades y de distintas disciplinas: Rafael González, PhD, profesor titular de la Pontificia Universidad Javeriana del Departamento de Ingeniería de Sistemas; Juan Sebastián Rozo, director de Asuntos Públicos para la región andina en $\mathrm{Ra}-$ ppi; Andrés Lombana, PhD, del Departamento de Comunicaciones de la Pontificia Universidad Javeriana, y Laura Cortés-Rico, PhD, de la Universidad Militar, quienes formularon interesantes puntos de vista frente al tema de la Sociedad 5.0.

Complementan el contenido de esta edición tres artículos con diferentes enfoques sobre el tema central de este número. El ingeniero Jeimy José Cano PhD, director y experto en Seguridad, escribe sobre las tecnologías emergentes en materia de Seguridad y Ciberseguridad para el 2030. Liliana Lindberg, MsC, ingeniera de Soluciones de IA, en
Swedish start-up Peltarion, se refiere a la Inteligencia Artificial, una de las más disruptivas tecnologías del siglo que puede incidir en el cambio de vida; presenta los retos de esta tecnología en algunos países y compañías de Europa. Así mismo, con Diana Lucio-Arias PhD, directora del Departamento de Ciencias de la Información de la Pontificia Universidad Javeriana, hacemos un resumen en el tiempo para observar de dónde vienen la Sociedad 5.0, esta revolución 5.0 y hacia dónde nos llevan, junto con los diversos cambios que se presentarán en la sociedad, en las organizaciones y en la educación.

Finalmente, me quiero referir a la situación que hoy aqueja al mundo. Para todos es claro que lo más importante es la vida del ser humano, todo lo que tenemos alrededor nos permite crecer como personas y profesionales, pero nada más tiene relevancia. Hoy que somos vulnerables frente a la pandemia, la solidaridad en función del cuidado colectivo es fundamental. ¡Cuidémonos para poder cuidar a otros! ¿Cómo cambiarán las prioridades del mundo después de esta pandemia? 数

Maria Mercedes Corral Strassmann, MsC. Ingeniero de Sistemas y Computación de la Universidad de Los Andes; Maestría en Comunicación de datos, University College London de la Universidad de Londres; Programa de Desarrollo Directivo - PDD de Inalde. Experiencia, como Director de Proyectos en el Banco de la República; Gerente de TI de CIFIN - Asobancaria; Vicepresidente de Tecnología de Deceval. Experiencia de más de 20 años como profesora universitaria en áreas de Ingeniería de software, y Gerencia de proyectos, Maestría y Especialización de Ingeniería de Sistemas en la Universidad Javeriana. En la actualidad es estudiante del Doctorado de Comunicación, Lenguajes e Información en la Universidad Javeriana. 